Research Article

\title{
Nomogram for Predicting Recurrence-Free Survival in Chinese Women with Endometrial Cancer after Initial Therapy: External Validation
}

\author{
Yuan Cheng, ${ }^{1}$ Yangyang Dong, ${ }^{1}$ Wenjuan Tian, ${ }^{2}$ Hua Zhang, ${ }^{3}$ Xiaoping Li, ${ }^{1}$ Zhiqi Wang, \\ Boer Shan, ${ }^{2}$ Yulan Ren, ${ }^{2}$ Lihui Wei, ${ }^{1}$ Huaying Wang $\mathbb{D}^{2},{ }^{2}$ and Jianliu Wang ${ }^{1}$ \\ ${ }^{1}$ Department of Obstetrics and Gynecology, Peking University People's Hospital, No. 11 Xizhimen South Street, Xicheng Dist., \\ Beijing 100044, China \\ ${ }^{2}$ Department of Gynecology, Fudan University Shanghai Cancer Center, No. 255, Dong'An Road, Shanghai 200032, China \\ ${ }^{3}$ Research Center of Clinical Epidemiology, Peking University Third Hospital, Xueyuan Rd 38\#, Haidian Dist., \\ Beijing 100191, China
}

Correspondence should be addressed to Huaying Wang; wanghuaying270@163.com and Jianliu Wang; wangjianliu@ pkuph.edu.cn

Received 11 September 2019; Revised 27 February 2020; Accepted 3 March 2020; Published 29 May 2020

Academic Editor: Francesca De Felice

Copyright (c) 2020 Yuan Cheng et al. This is an open access article distributed under the Creative Commons Attribution License, which permits unrestricted use, distribution, and reproduction in any medium, provided the original work is properly cited.

\begin{abstract}
This study aimed at developing an available recurrence-free survival (RFS) model of endometrial cancer (EC) for accurate and individualized prognosis assessment. A training cohort of 520 women with EC who underwent initial surgical treatment and an external validation cohort of 445 eligible EC patients from 2006 to 2016 were analyzed retrospectively. Multivariable Cox proportional hazards regression models were used to develop nomograms for predicting recurrence. The concordance index (Cindex) and the area under the receiver operating characteristic curve (AUC) were calculated to determine the discrimination of RFS prognostic scoring systems. Calibration plots were generated to examine the performance characteristics of the predictive nomograms. Regression analysis revealed that an advanced International Federation of Gynecology and Obstetrics (FIGO) stage, histological grade 3 , primary tumor diameter $\geq 2 \mathrm{~cm}$, and positive peritoneal cytology were independent prognostic factors for RFS in EC in the training set. The nomograms estimated RFS according to these four variables, with a C-index of 0.860 , which was superior to that of FIGO stage (2009 criteria), at $0.809(P=0.034)$, in the training cohort. Encouragingly, consistent results were observed in the validation set, with a C-index of 0.875 for the nomogram and a C-index of 0.833 for the FIGO staging $(P=0.0137)$. Furthermore, the calibrations of the nomograms predicting 3-and 5-year RFS strongly corresponded to the actual survival outcome. In conclusion, this study developed an available nomogram with effective external validation and relatively appreciable discrimination and conformity for the accurate assessment of 3- and 5-year RFS in Chinese women with EC.
\end{abstract}

\section{Introduction}

Endometrial cancer (EC) is the most common gynecological malignancy, ranking as the fourth among female tumors in developed countries [1]. Epidemiological analysis in China showed that the morbidity and mortality rates of EC have increased over recent years [2,3]. Endometrioid adenocarcinoma, also known as type $\mathrm{I}$, is the most frequent histological subtype and accounts for about $65 \%$. Nonendometrioid endometrial cancer which includes uterine serous carcinoma, clear cell cancer, and carcinosarcoma was identified as type II and accounts for about $35 \%$. The 5-year overall survival of type I and type II was about $85 \%$ and $55 \%$, respectively [4]. Although EC is detected early in most cases and patients begin receiving appropriate treatment with a good prognosis, the 5-year overall survival of patients with stage I-III EC ranges from $57 \%$ to $91 \%$ and with stage IV is $20-26 \%$ [5-7]. The prognosis of endometrial cancer is also affected by many other factors such as age, tumor grade, and positive peritoneal cytology 
[8]. Individual differences in recurrence or death in women with EC at 2 to 3 years after primary therapy vary widely $[9,10]$. Hence, it is urgent to place greater emphasis on precise and individualized prognosis evaluation and monitoring strategies for the management of patients with EC.

Individualized mathematical nomograms have been widely adopted as auxiliary tools to guide clinical decision making in medical fields [11-14]. In 2014, AlHilli et al. developed nomograms stratified histologically to predict the overall survival of EC patients [10]. In 2016, a nomogram predicted a low recurrence rate in women with EC (stages I-III), which could reduce unnecessary treatment by $60 \%$ [15]. However, these risk-scoring models have only been performed in analyses of internal data, and they lack external validation in independent samples based on established mathematical formulas.

This study retrospectively analyzed data from 965 women with stage I-IV EC from two large-scale hospitals that have focused on EC treatment in China over the last 10 years. A nomogram with good discrimination and calibration was developed for both internal and external validation cohorts based on clinicopathological characteristics to predict the probability of 3- and 5-year recurrence-free survival (RFS) and overall survival in women with EC.

\section{Materials and Methods}

2.1. Patients. The retrospective cohort study included 965 patients who underwent hysterectomy for stage I-IV EC in Peking University People's Hospital (training cohort, $n=520$ ) and Fudan University Shanghai Cancer Center (validation cohort, $n=445$ ) from January 2006 to December 2016. The present study was approved by the Ethics Committee of Peking University People's Hospital and Fudan University Shanghai Cancer Center. The exclusion criteria were incomplete clinical data or lost to follow-up. The baseline characteristics collected for all patients were as follows: (1) essential variables: age and menopausal status; (2) clinical and surgical variables: surgical procedure (with or without lymphadenectomy); (3) pathological variables: FIGO stage, pathological type, differentiation status, tumor size, peritoneal cytology status, lymphovascular space involvement (LVSI), lymph node metastasis, depth of myometrial invasion, and cervical stromal invasion (clinical stage and histological grade for all patients were classified in accordance with the 2009 FIGO criteria and pathological type followed by the two types of endometrial carcinoma of Bokhman in 1983); and (4) adjuvant therapy information: radiotherapy, chemotherapy, or their combination. Endometrial cancer patients with high-risk factors were performed adjuvant treatment after surgery according to pathological findings and comprehensive multidisciplinary discussion based on international guidelines. Generally, high-risk factors usually include age $\geq 60$, myometrial invasion $\geq 50 \%$, grade 3 , LVSI positive, and type II endometrial cancer. Patients without any high-risk factor were considered as in the low-risk group who just needed followup. Patients with high-risk factors (age $\geq 60$, grade 3, myometrial invasion $\geq 50 \%$, stage II, and LVSI) were recommended for radiotherapy or chemotherapy. If the patients with more high-risk factors (stage III-IV, type II endometrial cancer), they were undergone combination regimen (radiotherapy and chemotherapy).

2.2. Treatment and Follow-Up. All women with stage I-IV EC were enrolled if they had undergone initial surgical treatment, including total hysterectomy with bilateral salpingo-oophorectomy with or without systematic lymph node dissection (pelvic \pm para-aortic lymphadenectomy). Patients who were at high risk for cancer development and those with an advanced cancer stage underwent postoperative adjuvant radiotherapy, systemic chemotherapy, or their combination. Patients were followed up after initial surgery. And the occurrence of recurrence or death of the patients was recorded. Physical examination and diagnostic imaging tests were performed according to the findings.

\subsection{Statistical Analysis}

2.3.1. Definition of RFS. The clinical outcome was evaluated according to recurrence-free survival (RFS). The duration of follow-up for RFS was defined as the time from hysterectomy-based surgical treatment to the date of first recurrence or last follow-up if there was no recurrence. In addition, Kaplan-Meier cumulative survival probability was used in this study. Cumulative survival probability was calculated by multiplying probabilities for each prior relapse time [16].

2.3.2. Nomogram of Prediction Model. The clinical and pathological variables were evaluated for an association with RFS by univariate and multivariate Cox proportional hazards regression analyses. Associations are represented by the hazard ratio (HR) and corresponding 95\% confidence intervals (CIs) assessed from the model. Variables with $P<0.05$ were identified as independent risk factors for RFS and were retained in the final model. Furthermore, the selected high-risk variables were included in the Cox proportional hazards models of RFS. The risk coefficient of each factor was calculated and included in the equations of the individual prediction models for each patient and is presented as nomograms.

2.3.3. Validation of the Prediction Models. The discrimination ability of the prediction models was estimated using the Harrell C-index. The C-index was calculated by Cox regression models of 1000 random bootstrap resamples with the same sample size for assessing model accuracy [17]. The C-index ranges from 0.5 to 1 , with greater than 0.5 defined as having predictive power. Kaplan-Meier curves were plotted according to the bisection method for stratified management by the nomogram scores for the high- and low-risk groups. Calibration plots were examined by graphic charts for monitoring the average and maximal errors between the predicted 3- and 5-year probability of RFS and the actual outcome frequencies by the Kaplan-Meier method. The 
specificity and sensitivity of the models based on the nomogram compared with the 2009 FIGO stage for predicting RFS were evaluated by calculating the area under the receiver operating characteristic (ROC) curve (AUC).

2.3.4. Additional Statistical Analysis. The follow-up time was described using median, ranging from min to max; frequencies and proportions were used for categorical variables. The clinical features of the cohorts were analyzed using Student's $t$-test. $P<0.05$ was considered statistically significant. Data were collected using Microsoft Excel and converted to .sav files. All analyses were performed using SPSS v20.0 and R v2.15.0 with the Hmisc, rms, and Presence Absence packages.

\section{Results}

3.1. Training Cohort. We included 520 women with EC in the training cohort (Figure 1). The percentage of types I EC was $87.5 \%$. The distribution of women EC was $84.8 \%$ with stages I and II and $15.2 \%$ with stages III and stage IV. The number of women with low-grade and high-grade EC was $393(75.6 \%)$ and 127 (24.4\%), respectively. The clinicopathological characteristics of women are shown in Table 1. The median follow-up period for RFS was 53 months (range, 1-110); 46 women (8.8\%) relapsed, and 474 (91.2\%) showed no recurrence. The median time from initial therapy to recurrence was 12 months (range, 1-100). The mean (SD) 3and 5-year RFS was $92.0 \% \pm 1.3 \%$ and $90.1 \% \pm 1.6 \%$, respectively.

3.2. Prediction Nomogram. The clinicopathological characteristics of EC patients from the training sets with or without recurrence were analyzed (Supplementary Table 1). The results of the univariate and multivariate analyses revealed that four of the screened variables including advanced stage, G3, primary tumor diameter $\geq 2 \mathrm{~cm}$, and positive peritoneal cytology were independent prognostic factors in the training group (Table 2). The predictive nomograms were constructed based on the selected covariates to assess the probability of 3- and 5-year RFS in the training set (Figure 2). The incorporated mathematical formula of the nomograms involved FIGO stages II $(\mathrm{HR}=2.4 ; 95 \%$ CI: 0.9-6.7), III ( $\mathrm{HR}=4.2$; 95\% CI: 1.9-9.5), and IV $(\mathrm{HR}=15.1$; 95\% CI: 5.4-42.8), G3 (HR=4.2; 95\% CI: 2.1-8.4), tumor diameter $\geq 2 \quad(\mathrm{HR}=2.9 ; 95 \% \mathrm{CI}: 1.0-8.4)$, and positive peritoneal cytology (HR $=2.5 ; 95 \% \mathrm{CI}: 1.2-5.0)$, with further score transformation. According to the formulas for the nomograms, the total scores for each patient for 3- and 5year RFS could be easily and accurately calculated to individualize the prognosis.

3.3. Comparison with FIGO Stage. The discrimination ability of the nomograms was compared with that of FIGO stage. The C-index of the RFS nomogram was 0.860 (95\% CI: $0.797-0.923)$, which was superior to that of the 2009 FIGO classification, at 0.809 (95\% CI: $0.738-0.879 ; P=0.034)$ in

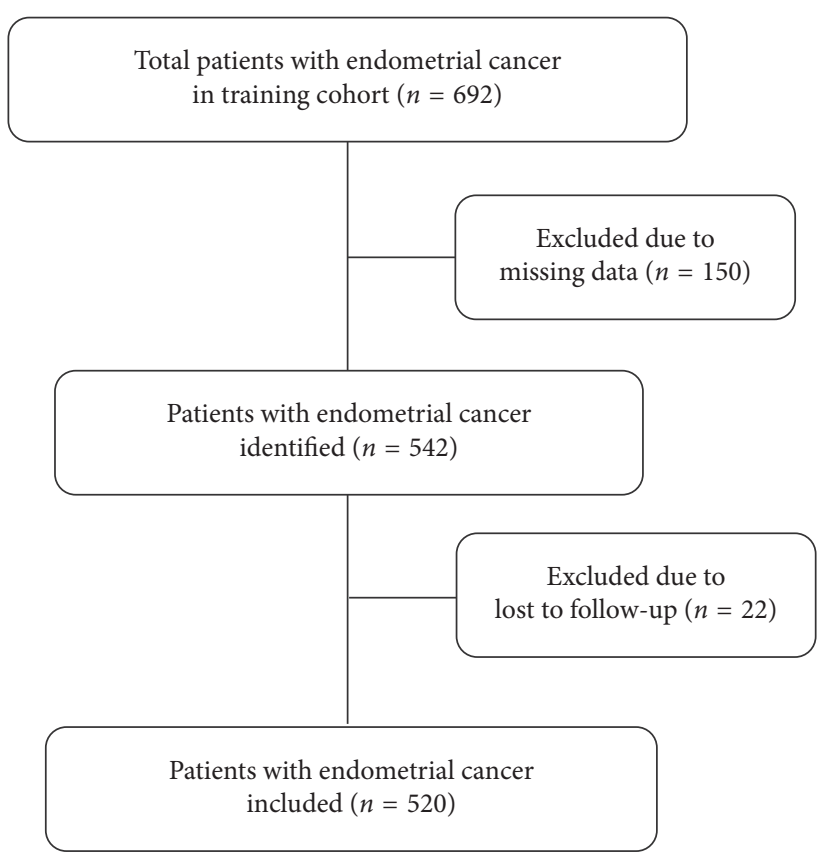

Figure 1: Flow diagram of the study participants.

the training set. Furthermore, the AUCs for the 3- and 5-year RFS nomograms were 0.894 (95\% CI: $0.832-0.956)$ and 0.873 (95\% CI: 0.812-0.934), respectively, which was superior to that of the 2009 FIGO classification at 0.849 (95\% CI: $0.777-0.920 ; P=0.0268$ ) and 0.816 (95\% CI: $0.75-0.89$; $P=0.0037$ ), respectively (Figures $3(\mathrm{a})$ and $3(\mathrm{~b})$ ).

3.4. Validation of the Nomogram. We recruited 445 eligible women with EC for the validation cohort. The clinicopathological characteristics of EC patients from the validation cohort with or without recurrence were analyzed (Supplementary Tables 2-4). The frequency of type I and type II EC was $359(80.7 \%)$ and 86 (19.3\%), respectively. The distribution of women with EC was as follows: 264 (59.3\%) with stage I, 38 (8.5\%) with stage II, 111 (25.0\%) with stage III, and $32(7.2 \%)$ with stage IV. The number of women with low-grade and high-grade EC was 297 (66.7\%) and 148 $(33.3 \%)$, respectively. The median follow-up period for RFS was 28 months (range, 1-112); 92 (20.7\%) women relapsed, and $353(79.3 \%)$ showed no recurrence. The median time from initial therapy to recurrence was 12 months (range, 1-63). The mean (SD) 3- and 5-year RFS was 78.8\% $\pm 1.2 \%$ and $72.8 \% \pm 1.6 \%$, respectively.

The 3- and 5-year RFS rates were also calculated in the validation cohort based on the nomogram for the training cohort. The C-index for the RFS nomogram was 0.875 (95\% CI: $0.829-0.921$ ), which was superior to that of the 2009 FIGO classification at 0.833 (95\% CI: $0.785-0.882$; $P=0.0137)$. The AUCs for 3- and 5-year RFS were 0.875 (95\% CI: 0.829-0.921) and 0.867 (95\% CI: 0.823-0.910), respectively, which were superior to those of the 2009 FIGO classification at 0.833 (95\% CI: $0.785-0.882 ; P=0.0137)$ and 0.829 (95\% CI: $0.785-0.882 ; \quad P=0.0296$ ), respectively (Figures 3(c) and 3(d)). 
TABLE 1: The clinicopathological characteristics of the training cohort.

\begin{tabular}{|c|c|}
\hline Variables & $\begin{array}{l}\text { Training cohort, } n=520 \\
\text { (no. of patients) }(\%)\end{array}$ \\
\hline \multicolumn{2}{|l|}{ Age (years) } \\
\hline$<60$ & $339(65.2)$ \\
\hline$\geq 60$ & $181(34.8)$ \\
\hline \multicolumn{2}{|l|}{ Menopausal status } \\
\hline No & $177(34.0)$ \\
\hline Yes & $343(66.0)$ \\
\hline \multicolumn{2}{|l|}{ Depth of myometrial invasion } \\
\hline$<50 \%$ & $391(75.2)$ \\
\hline$\geq 50 \%$ & $129(24.8)$ \\
\hline \multicolumn{2}{|l|}{ Cervical stromal invasion } \\
\hline No & $461(88.7)$ \\
\hline Yes & $59(11.3)$ \\
\hline \multicolumn{2}{|l|}{ Adnexal involvement } \\
\hline No & $492(94.6)$ \\
\hline Yes & $28(5.4)$ \\
\hline \multicolumn{2}{|l|}{ FIGO stage } \\
\hline I & $404(77.7)$ \\
\hline II & $37(7.1)$ \\
\hline III & $62(11.9)$ \\
\hline IV & $17(3.3)$ \\
\hline \multicolumn{2}{|l|}{ Histological grade } \\
\hline Grade 1 & $161(31.0)$ \\
\hline Grade 2 & $232(44.6)$ \\
\hline Grade 3 & $127(24.4)$ \\
\hline \multicolumn{2}{|l|}{ Histological type } \\
\hline Type I & $455(87.5)$ \\
\hline Type II & $65(12.5)$ \\
\hline \multicolumn{2}{|l|}{ Tumor diameter } \\
\hline$<2 \mathrm{~cm}$ & $189(36.3)$ \\
\hline$\geq 2 \mathrm{~cm}$ & $331(63.7)$ \\
\hline \multicolumn{2}{|l|}{ Peritoneal cytology } \\
\hline No & $473(91.0)$ \\
\hline Yes & $47(9.0)$ \\
\hline \multicolumn{2}{|l|}{ LVSI } \\
\hline No & $331(74.4)$ \\
\hline Yes & $114(25.6)$ \\
\hline \multicolumn{2}{|l|}{ Lymph node involvement } \\
\hline No & $401(77.1)$ \\
\hline Yes & $49(9.4)$ \\
\hline Unknown & $70(13.5)$ \\
\hline \multicolumn{2}{|l|}{ Lymphadenectomy } \\
\hline No & $70(13.5)$ \\
\hline Yes & $450(86.5)$ \\
\hline \multicolumn{2}{|l|}{ Adjuvant therapy } \\
\hline No adjuvant therapy & $285(54.8)$ \\
\hline Radiotherapy & $10(2.0)$ \\
\hline Chemotherapy & $165(31.7)$ \\
\hline Radiotherapy + cChemotherapy & $60(11.5)$ \\
\hline \multicolumn{2}{|l|}{ Recurrence } \\
\hline No & $474(91.2)$ \\
\hline Yes & $46(8.8)$ \\
\hline \multicolumn{2}{|l|}{ Follow-up (months) } \\
\hline Median & 53 \\
\hline Mean & 48.7 \\
\hline Range & $1-110$ \\
\hline
\end{tabular}

Data are expressed as $n(\%)$ or the means \pm SD. FIGO $=$ International Federation of Gynecology and Obstetrics; LVSI = lymphovascular space involvement.
Calibration plots for the nomograms to predict 3- and 5year RFS were calculated in the internal validation. The predicted 3- and 5-year RFS rates were similar to the actual survival rates, with small average error rates of less than $10 \%$ and a lack of bias, as represented by the dotted lines in Figures 4(a) and 4(b). Moreover, external validation showed no dramatic differences between the predicted and actual 3and 5-year RFS rates (Figures 4(c) and 4(d)).

\subsection{Optimal Nomogram Threshold and Redistribution.}

The low- and high-risk groups were defined according to the optimal threshold of the ROC calculated from the recurrence distribution of each probability of the RFS nomograms in the training cohort $(P=0.029)$. We further analyzed the distribution of patients in the low- and high-risk groups estimated by the nomogram scores. The frequency of lowand high-risk EC was 371 (71.3\%) and 149 (28.7\%), respectively (Table 3 ). We found individual differences in recurrence in women with EC after redistribution. In the low-risk group, the characteristic distribution among EC patients with high-risk factors was as follows: advanced stage $(4,1.1 \%)$, grade $3(22,5.9 \%)$, primary tumor diameter $\geq 2 \mathrm{~cm}$ $(192,51.8 \%)$, and positive peritoneal cytology $(1,0.3 \%)$. In the high-risk group, the number of patients with low-risk factors was as follows: FIGO stage I, G1/G2, primary tumor diameter $<2 \mathrm{~cm}$, and negative peritoneal cytology was 55 (36.9\%), 44 (29.5\%), 10 (6.7\%), and 103 (69.1\%), respectively.

\section{Discussion}

Predictive nomograms for the assessment of EC prognosis and recurrence have preliminarily been well developed in Europe and the United States $[10,15]$. Nevertheless, these prediction models are heterogeneous according to women presenting with EC in different populations [18]. As far as we know, a nomogram to predict recurrence in EC patients based on the Chinese population has not been established until now. In the current study, we developed a nomogram to predict recurrence in women with EC in China, which was well validated in an independent cohort from Fudan University Shanghai Cancer Center. An available nomogram for predicting RFS in Chinese women with EC after initial therapy was preliminarily developed and externally validated. We have sorted out a table to compare the predicting recurrence-free survival model with previous studies (Table 4). (1) The predictive model was established based on the data from patients with endometrial cancer in all stages (I-IV), not just focusing on early stages (I-III) in this study. (2) The independent risk factors of endometrial cancer recurrence selected by multivariate analysis in the present study were some differences from those in the previous studies. Advanced stage, grade 3, primary tumor diameter $\geq 2 \mathrm{~cm}$, and positive peritoneal cytology were independent recurrent factors in the training group in this study. One or more of these indicators have been included in previously 
TABLE 2: Multivariate Cox proportional hazards regression analysis for recurrence-free survival (RFS) in the training cohort.

\begin{tabular}{|c|c|c|c|c|}
\hline Variables & $\begin{array}{l}\text { Univariate analysis } \\
\text { OR }(95 \% \mathrm{CI})\end{array}$ & $P$ value & $\begin{array}{c}\text { Multivariate analysis } \\
\text { Adjusted OR (95\% CI) }\end{array}$ & $P$ value \\
\hline \multicolumn{5}{|l|}{ Age (years) } \\
\hline$<60$ & 1 (referent) & & & \\
\hline$\geq 60$ & $2.4(1.3-4.3)$ & 0.003 & & \\
\hline \multicolumn{5}{|l|}{ Depth of myometrial invasion } \\
\hline$<50 \%$ & 1 (referent) & & & \\
\hline$\geq 50 \%$ & $2.9(1.6-5.2)$ & $<0.001$ & & \\
\hline \multicolumn{5}{|l|}{ Cervical stromal invasion } \\
\hline No & 1 (referent) & & & \\
\hline Yes & $4.1(2.2-7.6)$ & $<0.001$ & & \\
\hline \multicolumn{5}{|l|}{ Histological grade } \\
\hline Grade $1 / 2$ & 1 (referent) & & 1 (referent) & \\
\hline Grade 3 & $9.4(5.0-18.0)$ & $<0.001$ & $4.2(2.1-8.4)$ & $<0.001$ \\
\hline \multicolumn{5}{|l|}{ Histological type } \\
\hline Type I & 1 (referent) & & & \\
\hline Type II & $8.5(4.7-15.2)$ & $<0.001$ & & \\
\hline \multicolumn{5}{|l|}{ Tumor diameter } \\
\hline$<2 \mathrm{~cm}$ & 1 (referent) & & 1 (referent) & \\
\hline$\geq 2 \mathrm{~cm}$ & $6.6(2.4-18.4)$ & $<0.001$ & $2.9(1.0-8.4)$ & 0.049 \\
\hline \multicolumn{5}{|l|}{ LVSI } \\
\hline No & 1 (referent) & & & \\
\hline Yes & $4.1(2.2-7.5)$ & $<0.001$ & & \\
\hline \multicolumn{5}{|l|}{ FIGO stage } \\
\hline I & 1 (referent) & & 1 (referent) & \\
\hline II & $5.4(2.0-14.3)$ & 0.001 & $2.4(0.9-6.7)$ & 0.099 \\
\hline III & $8.8(4.1-19.0)$ & $<0.001$ & $4.2(1.9-9.5)$ & $<0.001$ \\
\hline IV & $84.3(36.3-195.5)$ & $<0.001$ & $15.1(5.4-42.8)$ & $<0.001$ \\
\hline \multicolumn{5}{|l|}{ Peritoneal cytology } \\
\hline No & 1 (referent) & & 1 (referent) & \\
\hline Yes & $10.1(5.6-18.3)$ & $<0.001$ & $2.5(1.2-5.0)$ & 0.013 \\
\hline \multicolumn{5}{|l|}{ Lymph node involvement } \\
\hline No & 1 (referent) & & & \\
\hline Yes & $8.1(4.2-15.5)$ & $<0.001$ & & \\
\hline \multicolumn{5}{|l|}{ Lymphadenectomy } \\
\hline No & 1 (referent) & & & \\
\hline Yes & $0.7(0.3-1.4)$ & 0.27 & & \\
\hline \multicolumn{5}{|l|}{ Adjuvant therapy } \\
\hline No adjuvant therapy & 1 (referent) & & & \\
\hline Radiotherapy & $9.6(2.0-45.6)$ & 0.004 & & \\
\hline Chemotherapy & $6.7(3.0-14.7)$ & $<0.001$ & & \\
\hline Radiotherapy + chemotherapy & $5.3(2.0-14.1)$ & $<0.001$ & & \\
\hline
\end{tabular}

$\mathrm{OR}=$ odds ratio; $\mathrm{CI}=$ confidence interval; LVSI = lymphovascular space involvement; FIGO = International Federation of Gynecology and Obstetrics.

established models; however, the combination of these four indicators was included in a recurrence model for the first time in this study. (3) Other studies have only predicted 3-year relapse-free survival in patients with endometrial cancer. Our prediction model is focused on both 3-year relapse-free survival and 5-year relapse-free survival. The model established in this study proved to be accurate and stable through external verification. The accuracy and the verification of the model were higher than those of other types of models. (4) We also compared our prediction model with the FIGO staging and found that it was superior to the FIGO staging in the prediction of 3-year or 5-year relapse-free survival of patients with endometrial cancer. This comparison has not been performed in previous studies.

Four independent risk factors, i.e., FIGO stage, histological grade, tumor diameter, and peritoneal cytology status, were primarily deemed predictive factors of RFS to develop a nomogram for patients with stage I-IV EC in the training set after confounding factors adjustment. 455 cases of type I and 65 cases of type II were included in this study. However, pathological type was not selected as an independent risk factor for recurrence in patients with endometrial cancer. Accumulating evidence has affirmed that 


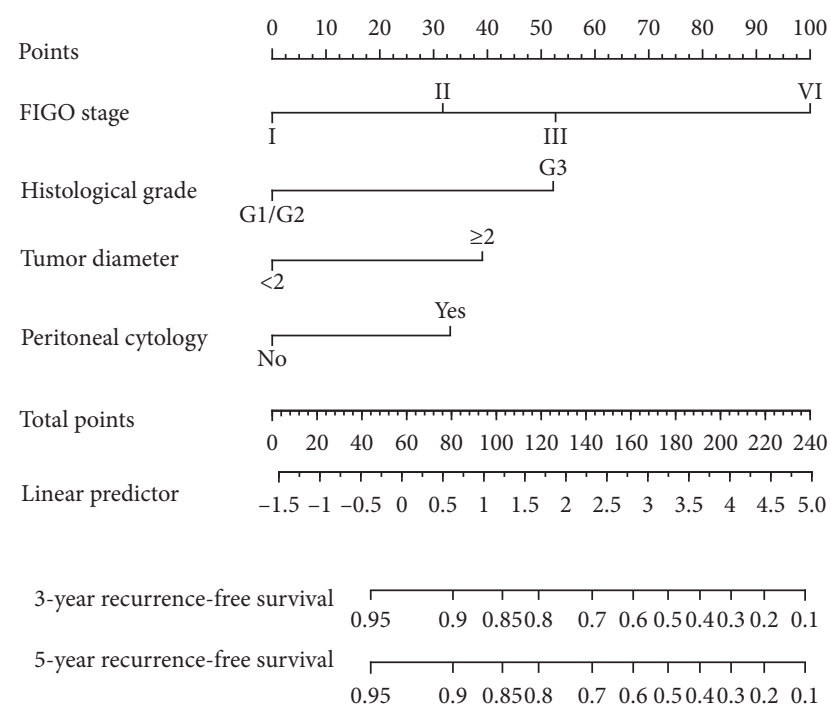

FIGURE 2: Nomograms for predicting 3- and 5-year recurrence-free survival (RFS) in patients with endometrial cancer. In order to evaluate the recurrence-free survival rate of each patient, the score of each variable was calculated by the value of the "Points" axis, and the sum of the values of all variables was corresponding to the number of the "Total points" axis. The vertical line of the total score was corresponding to 3- and 5-year probability of recurrence-free survival.

LVSI significantly contributes to replapse in stage (I-III) and high-risk EC patients $[9,14]$. However, LVSI was not the most appropriate pathological variable for assessing recurrence risk in our study, which may be due to the limited sample size.

The influence of the peritoneal cytology status on the prognosis of women with EC remains and should be further evaluated. Previous multivariate analyses have revealed that positive peritoneal cytology could predict relapse and tumor-related death in early-stage EC [19-23]. However, other studies found that, in low-risk patients with EC, positive peritoneal cytology did not affect the 5year disease-free survival rate $[24,25]$. Positive peritoneal cytology has also previously shown no effects on overall survival or disease-free survival in patients with low- or intermediate-risk disease $[26,27]$. In the present study, we found that positive peritoneal cytology was a critical and independent prognostic factor of EC recurrence in both cohorts. The possible reason may be that our study involved EC patients with progressive staging or poor differentiation.

Notably, the results showed that the RFS estimation of the nomograms according to the four variables was superior to that of the 2009 FIGO classification in the training cohort. The calibrations of the nomograms predicting 3- and 5-year RFS highly corresponded to the actual survival rates, with minute average error rates of less than $10 \%$ for both. We further divided the patients into low- and high-risk groups according to the optimal threshold of ROC from the recurrence distribution of each probability of the RFS nomograms. Predictably, we discovered women presenting only one high-risk factor who were represented in the low- risk group and women with low-stage disease presenting other high-risk factors allocated to the high-risk group. Therefore, individual differences in prognosis are more common in EC patients.

Kondalsamy-Chennakesavan et al. developed nomograms to predict EC recurrence in 2097 patients with stage I-III EC from 1997 to 2009. The multivariate Cox model indicated that age, FIGO stage, histological grade, LVSI, tumor type, and peritoneal cytology status were independent prognostic factors of EC relapse [28]. Bendifallah et al. attempted to validate the Kondalsamy-Chennakesavan nomogram of EC for prognosis evaluation in 271 cases with stage I-III EC using an independent, multicenter external patient cohort. However, results showed that the nomogram was only partially generalized in another independent population, with a discrimination ability of 0.66 for 3-year recurrence-free survival [18]. The study reported disparate population characteristics, recommendations for lymph node resection, and adjuvant chemotherapy in multiple regions, and the efficacy of the prediction model in the external validation was relatively limited. Further optimization and improvement of nomograms and existing risk stratification strategies are needed [29]. In the present study, based on the four variables of the model, we verified the accuracy of our nomogram in an external cohort, with a C-index of 0.875 for the predicted 3-year RFS of EC. Additionally, the calibration curve was acceptable despite little differences in several clinical characteristics, recurrence outcomes, and follow-up times between the two populations. The number of patients with advanced endometrial cancer and the proportion of patients with recurrence in the validation cohort were higher than those in the training cohort. However, the follow-up time of EC patients in the validation cohort was shorter. More optimistically, the above four covariates used to develop the prediction models in the training cohort were also identified as independent prognostic factors of EC relapse in the validation cohort by multivariate analysis.

Our study has some limitations. Retrospective clinical data with uncertain potential confounding factors could negatively affect the accuracy of the results. Inevitably, many observations with missing data were deleted, which could have caused bias. Abu-Rustum et al. included the number of lymph nodes removed during comprehensive surgery in a predictive model of prognosis for women with EC [14]. AlHilli et al. revealed inadequate/negative lymphadenectomy as an independent risk factor in low-risk patients with EC [10]. We also assessed the value of surgical treatment and adjuvant therapy in the recurrence of patients with EC. Univariate analysis showed that they were associated with endometrial cancer recurrence. However, multivariate analysis showed that they were not independent risk factors for endometrial cancer recurrence in our study. The reason may be the limited number of cases or other interaction factors. Additionally, the modeling and validation groups differed in clinical characteristics and recurrence rates, which might also have affected the results. Finally, further optimization of this model in a national multicenter study is needed. 


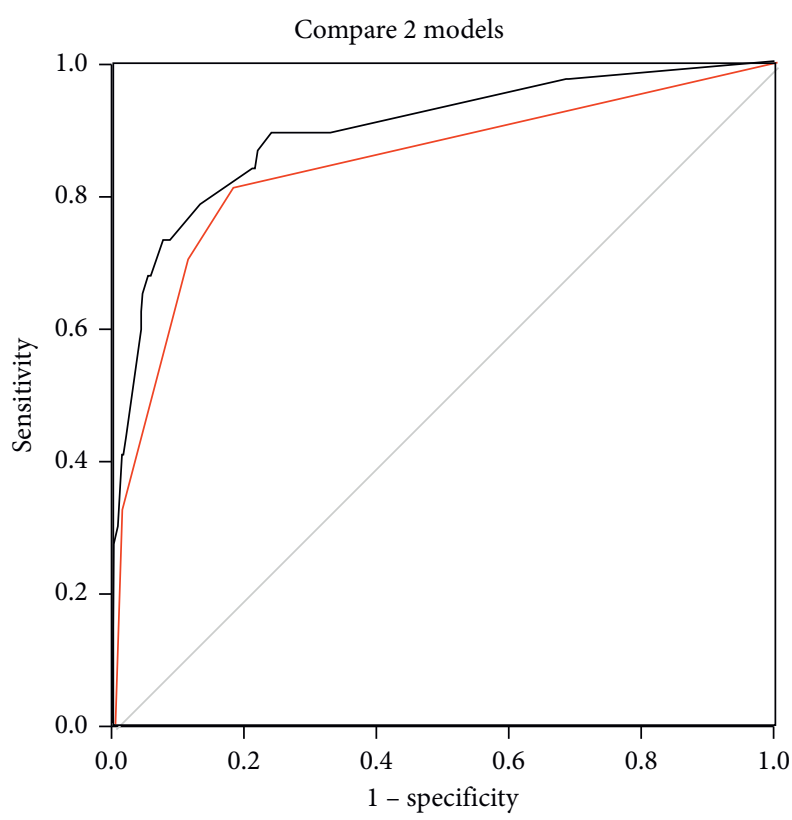

AUC

— Model 1: 0.894

— Model 2: 0.849

(a)

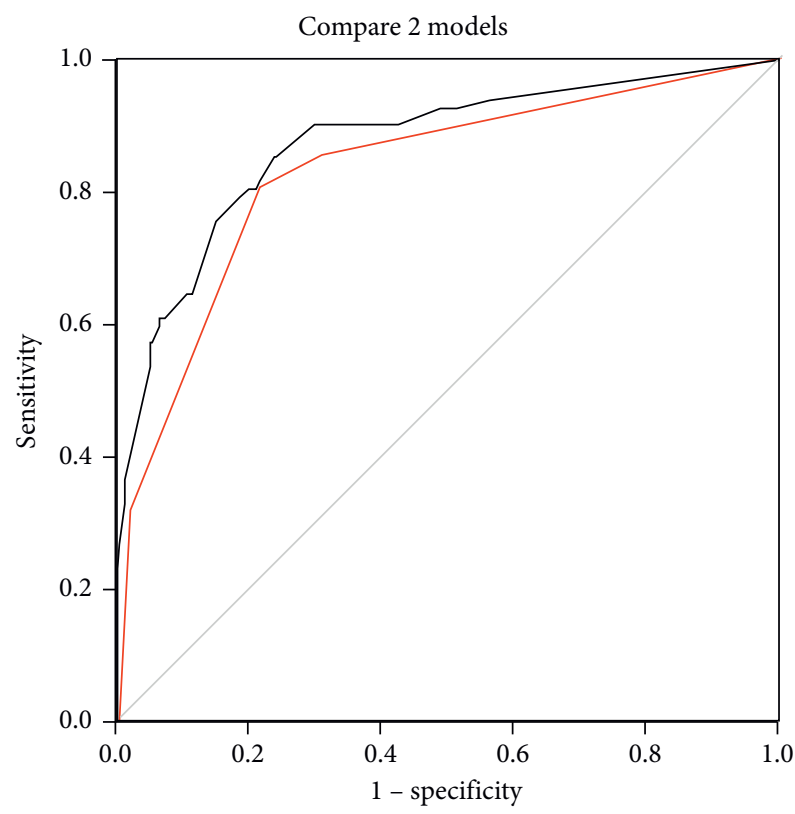

AUC

Model 1: 0.875

Model 2: 0.833

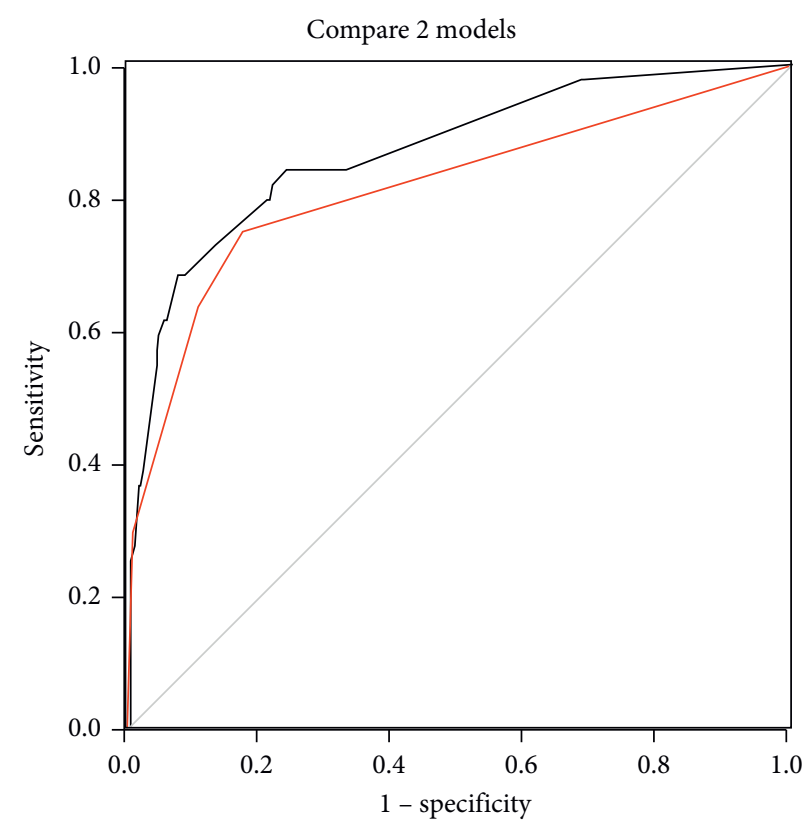

AUC

— Model 1: 0.873

_ Model 2: 0.816

(b)

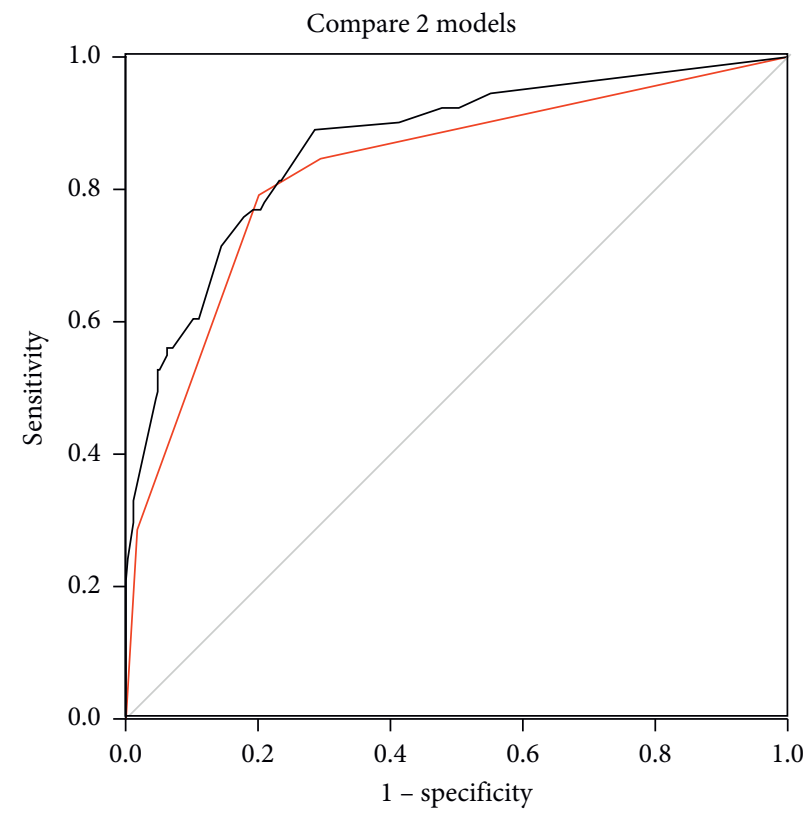

AUC

— Model 1: 0.867

Model 2: 0.829

(c)

(d)

Figure 3: Area under the receiver operating characteristic curve (AUC) values of the nomogram and the 2009 International Federation of Gynecology and Obstetrics (FIGO) stage system for the training and validation cohorts to assess the 3- and 5-year recurrence-free survival (RFS). ( $a, b)$ Comparing the AUCs of nomogram with FIGO stage in the training group (T) for predicting 3-and 5-year recurrence-free survival (RFS) in patients with endometrial cancer. (c, d) Comparing the AUCs of nomogram with FIGO stage in the validation group (V) for predicting 3- and 5-year recurrence-free survival (RFS) in patients with endometrial cancer. Model 1 of black line represents nomogram; model 2 of red line represents FIGO stage. (a) T: 3 RFS-AUC nomogram:FIGO, (b) T: 5 RFS-AUC nomogram:FIGO, (c) V: 3 RFS-AUC nomogram:FIGO, and (d) V: 5 RFS-AUC nomogram:FIGO. 


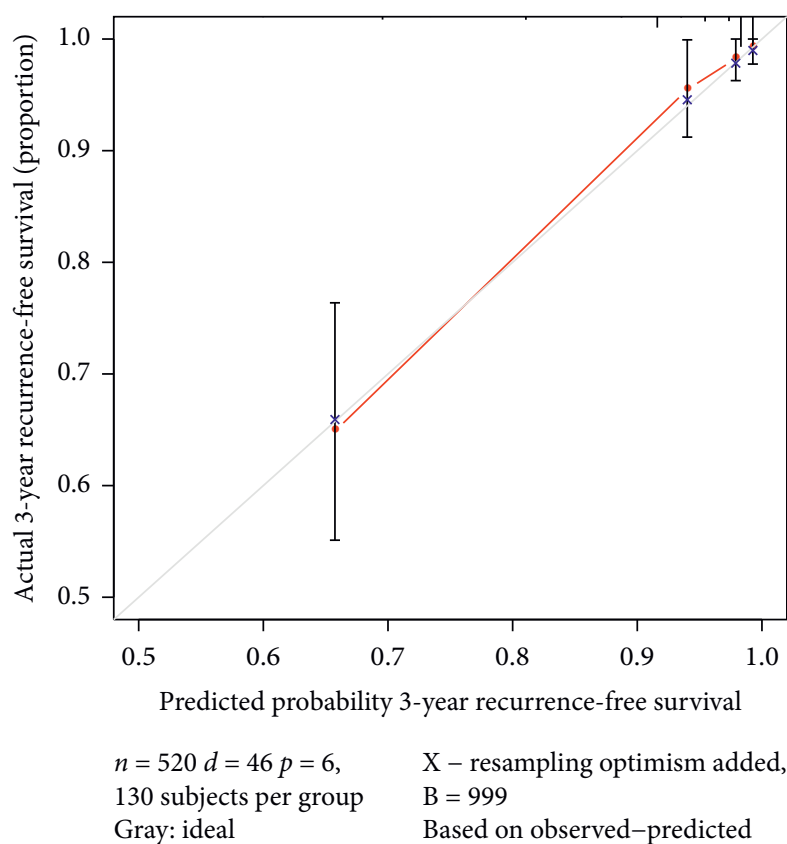

(a)

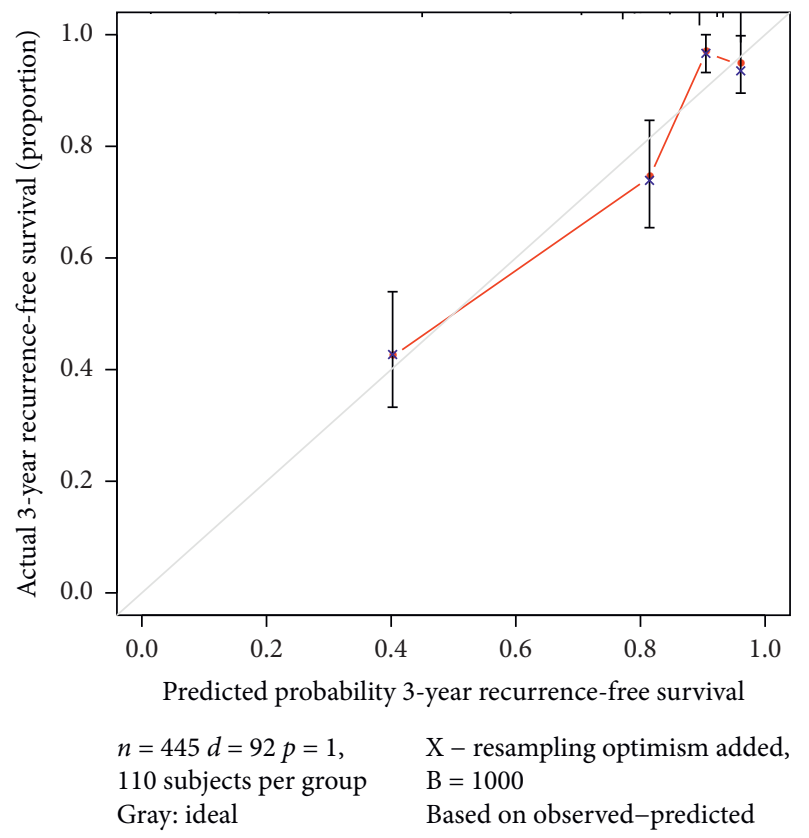

(c)

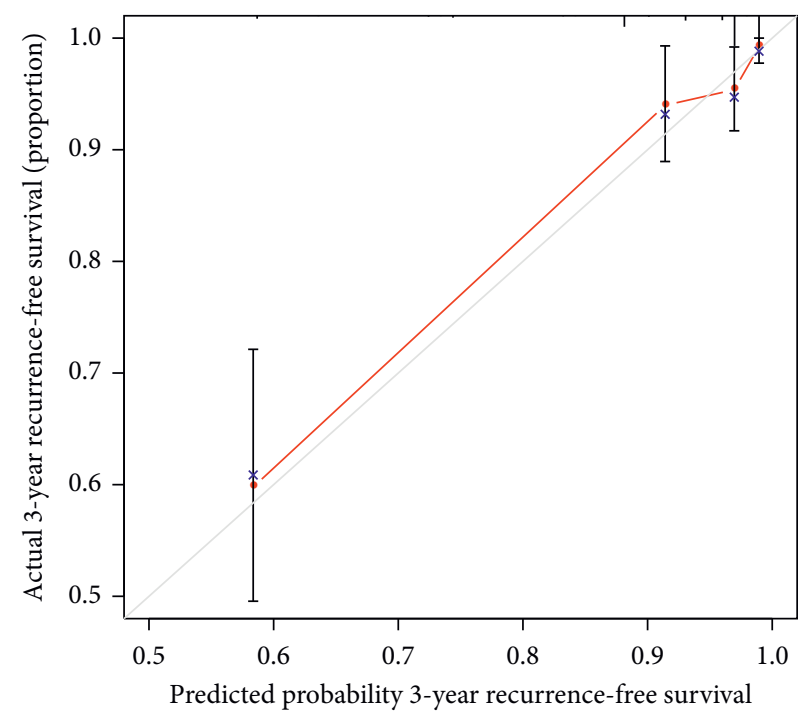

$\begin{array}{ll}n=520 d=46 p=6, & \mathrm{X}-\text { resampling optimism added, } \\ 130 \text { subjects per group } & \mathrm{B}=1000 \\ \text { Gray: ideal } & \text { Based on observed-predicted }\end{array}$

(b)

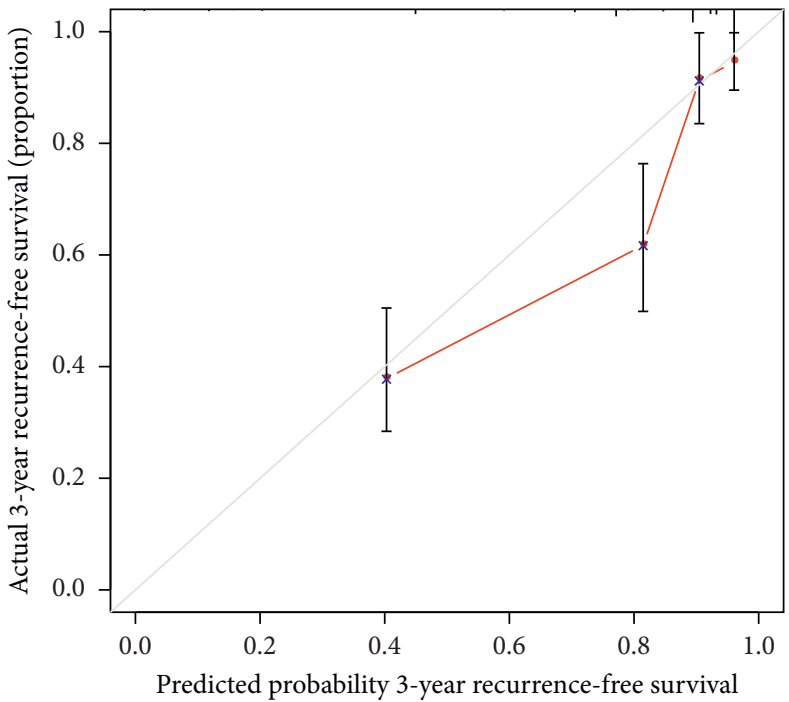

$n=445 d=92 p=1, \quad \mathrm{X}-$ resampling optimism added, 110 subjects per group $\quad \mathrm{B}=1000$

Gray: ideal
Based on observed-predicted

FIGURE 4: Calibration of the nomograms for 3- and 5-year recurrence-free survival (RFS) in patients with stage I-IV endometrial cancer in the training and validation cohorts. $X$-axis indicates the predicting probability of nomogram. $Y$-axis shows the actual 3- or 5-year probability of survival as assessed by Kaplan-Meier curves. Red line represents the predicted probability of nomogram. Gray line represents ideal consistency between the prediction and actual probabilities of 3- or 5-year RFS. Vertical bars represent 95\% CI. Blue dots correspond to the accuracy of the prediction. (a, b) Calibrations of the nomogram in the training cohort for predicting 3- and 5-year RFS. (c, d) Calibrations of the nomogram in the validation cohort for predicting 3- and 5-year RFS.

We developed a clinically available and relatively precise model based on nomograms to predict RFS in Chinese women with EC. The study specifically incorporated the four independent prognosis covariates of advanced stage, grade 3 , tumor diameter $\geq 2 \mathrm{~cm}$, and positive peritoneal cytology and helped develop a tool that may be conducive for developing individualized therapeutic strategies for Chinese patients with EC. 
TABLE 3: The distribution of patients in the low- and high-risk cohorts estimated by the nomogram scores in the training cohort.

\begin{tabular}{|c|c|c|c|}
\hline Variables & Low risk, $N=371(71.3 \%)$ & High risk, $N=149(28.7 \%)$ & $P$ value \\
\hline FIGO stage & & & $<0.001$ \\
\hline I & $349(94.1)$ & $55(36.9)$ & \\
\hline II & $18(4.8)$ & $19(12.8)$ & \\
\hline III & $4(1.1)$ & $58(38.9)$ & \\
\hline IV & $0(0)$ & $17(11.4)$ & \\
\hline Histological grade & & & $<0.001$ \\
\hline Grade $1 / 2$ & $349(94.1)$ & $144(29.5)$ & \\
\hline Grade 3 & $22(5.9)$ & $105(70.5)$ & \\
\hline Primary tumor diameter & & & $<0.001$ \\
\hline$<2 \mathrm{~cm}$ & $179(48.2)$ & $10(6.7)$ & \\
\hline$\geq 2 \mathrm{~cm}$ & $192(51.8)$ & $139(93.3)$ & \\
\hline Peritoneal cytology & & & $<0.001$ \\
\hline No & $370(99.7)$ & $103(69.1)$ & \\
\hline Yes & $1(0.3)$ & $146(30.9)$ & \\
\hline
\end{tabular}

Data are expressed as $n$ (\%). FIGO = International Federation of Gynecology and Obstetrics.

TABLE 4: Comparison of this study with previous studies of predictive models for recurrence-free survival of endometrial cancer patients.

\begin{tabular}{|c|c|c|c|c|}
\hline & Obermair A & Bendifallah S & Ouldamer L & Wang J \\
\hline Number of cases & 2097 & 396 & 861 & 520 \\
\hline Recurrence-free survival & 3-year & 3-year & 3-year & 3- and 5-year \\
\hline Histologic type & I, II & I & I, II & I, II \\
\hline FIGO stage & I-III & I-III & I-III & I-IV \\
\hline \multicolumn{5}{|l|}{ Factors } \\
\hline Age & Yes & Yes & Yes & - \\
\hline Histological grade & Yes & Yes & - & Yes \\
\hline FIGO stage & Yes & - & Yes & Yes \\
\hline Histologic type & Yes & - & Yes & - \\
\hline Tumor diameter $\geq 2 \mathrm{~cm}$ & - & Yes & - & Yes \\
\hline Myometrial invasion $\geq 50 \%$ & - & Yes & - & - \\
\hline LVSI & Yes & Yes & Yes & - \\
\hline Peritoneal washing & Yes & - & - & Yes \\
\hline Surgical nodal staging & - & - & Yes & \\
\hline Comparison with FIGO stage & - & - & - & Superior \\
\hline 3 -year internal validation & Yes $(0.86)$ & Yes $(0.74)$ & Yes $(0.75)$ & Yes $(0.89)$ \\
\hline 5-year internal validation & - & - & - & Yes $(0.87)$ \\
\hline 3-year external validation & - & Yes $(0.82)$ & - & Yes $(0.88)$ \\
\hline 5-year external validation & - & - & - & Yes $(0.87)$ \\
\hline
\end{tabular}

FIGO = International Federation of Gynecology and Obstetrics; LVSI = lymphovascular space involvement.

\section{Abbreviations}

RFS: $\quad$ Recurrence-free survival

EC: $\quad$ Endometrial cancer

C-index: Concordance index

AUC: $\quad$ Area under the receiver operating characteristic curve

FIGO: Federation of Gynecology and Obstetrics

EC: $\quad$ Endometrial cancer

LVSI: Lymphovascular space involvement

HR: $\quad$ Hazard ratio.

\section{Data Availability}

The datasets used and/or analyzed during the current study are available from the corresponding author on reasonable request.

\section{Conflicts of Interest}

The authors declare that they have no conflicts of interest.

\section{Authors' Contributions}

Yuan Cheng and Yangyang Dong contributed equally to this article. Prof. Jianliu Wang and Prof. Huaying Wang have substantial contributions to the conception and design of this project. Yuan Cheng and Yangyang Dong were responsible for the acquisition of data, or analysis and interpretation of data, and writing and revising the manuscript. Hua Zhang mainly gave guidance in data statistics and $\mathrm{R}$ language programming. Wenjuan Tian, Boer Shan, and Yulan Ren mainly established the database of endometrial cancer from Fudan University Shanghai Cancer Center. Prof. Zhiqi Wang, Prof. Xiaoping Li, and Prof. Lihui 
Wei gave good advice for the clinical application of this predicting model.

\section{Acknowledgments}

This study was supported by the National Natural Science Foundation of China (Grant no. 81802607), Special Projects for Strengthening Basic Research of Peking University (Grant no. BMU2018JC005), the Fund for Fostering Young Scholars of Peking University Health Science Center (Grant no. BMU2017PY011), the Research and Development Fund of Peking University People's Hospital (Grant no. RDY201712), and National Key Technology Research and Development Program of the Ministry of Science and Technology of China (Grant no. 2015BAI13B06).

\section{Supplementary Materials}

Supplementary Table 1: clinicopathological characteristics of 520 endometrial cancer patients from the training cohort with or without recurrence. Supplementary Table 2: the clinicopathological characteristics of 445 endometrial cancer patients of the validation cohort. Supplementary Table 3: characteristics of 445 women with endometrial carcinoma in the validation cohort with or without recurrence. Supplementary Table 4: multivariate Cox proportional hazards regression analysis for recurrence-free survival (RFS) in the validation cohort. (Supplementary Materials)

\section{References}

[1] R. L. Siegel, K. D. Miller, and A. Jemal, "Cancer statistics, 2018,” CA: A Cancer Journal for Clinicians, vol. 68, no. 1, pp. 7-30, 2018.

[2] W. Chen, R. Zheng, P. D. Baade et al., "Cancer statistics in China, 2015," CA: A Cancer Journal for Clinicians, vol. 66, no. 2, pp. 115-132, 2016.

[3] W. Chen, K. Sun, R. Zheng et al., "Cancer incidence and mortality in China, 2014," Chinese Journal of Cancer Research, vol. 30 , no. 1, pp. 1-12, 2018.

[4] J. V. Bokhman, "Two pathogenetic types of endometrial carcinoma," Gynecologic Oncology, vol. 15, no. 1, pp. 10-17, 1983.

[5] P. Morice, A. Leary, C. Creutzberg, N. Abu-Rustum, and E. Darai, "Endometrial cancer," The Lancet, vol. 387, no. 10023, pp. 1094-1108, 2016.

[6] M. Aamir Sheikh, A. D. Althouse, K. E. Freese et al., "USA endometrial cancer projections to 2030: should we be concerned?” Future Oncology, vol. 10, no. 16, pp. 2561-2568, 2014.

[7] S. Bendifallah, L. Ouldamer, V. Lavoue et al., "Patterns of recurrence and outcomes in surgically treated women with endometrial cancer according to ESMO-ESGO-ESTRO Consensus Conference risk groups: results from the FRANCOGYN study Group," Gynecologic Oncology, vol. 144, no. 1, pp. 107-112, 2017.

[8] Y. Dai, Y. Dong, Y Cheng et al., "Prognostic significance of lymphovascular space invasion in patients with endometrioid endometrial cancer: a retrospective study from a single center," Journal of Gynecologic Oncology, vol. 31, 2019.

[9] S. Bendifallah, G. Canlorbe, P. Collinet et al., "Just how accurate are the major risk stratification systems for early-stage endometrial cancer?" British Journal of Cancer, vol. 112, no. 5, pp. 793-801, 2015.

[10] M. M. AlHilli, A. Mariani, J. N. Bakkum-Gamez et al., "Riskscoring models for individualized prediction of overall survival in low-grade and high-grade endometrial cancer," $G y$ necologic Oncology, vol. 133, no. 3, pp. 485-493, 2014.

[11] Y. Dong, Y. Cheng, W Tian et al., "An externally validated nomogram for predicting lymph node metastasis of presumed stage I and II endometrial cancer," Frontiers in Oncology, vol. 9, p. 1218, 2019.

[12] Y. Kim, G. A. Margonis, J. D. Prescott et al., "Nomograms to predict recurrence-free and overall survival after curative resection of adrenocortical carcinoma," JAMA Surgery, vol. 151, no. 4, pp. 365-373, 2016.

[13] R. Rouzier, C. Uzan, A. Rousseau et al., "Multicenter prospective evaluation of the reliability of the combined use of two models to predict non-sentinel lymph node status in breast cancer patients with metastatic sentinel lymph nodes: the MSKCC nomogram and the Tenon score. Results of the NOTEGS study," British Journal of Cancer, vol. 116, no. 9, pp. 1135-1140, 2017.

[14] N. R. Abu-Rustum, Q. Zhou, J. D. Gomez et al., "A nomogram for predicting overall survival of women with endometrial cancer following primary therapy: toward improving individualized cancer care," Gynecologic Oncology, vol. 116, no. 3, pp. 399-403, 2010.

[15] L. Ouldamer, S. Bendifallah, G. Body et al., "Predicting poor prognosis recurrence in women with endometrial cancer: a nomogram developed by the FRANCOGYN study group," British Journal of Cancer, vol. 115, no. 11, pp. 1296-1303, 2016.

[16] A. Hazra and N. Gogtay, "Biostatistics series module 9: survival analysis," Indian Journal of Dermatology, vol. 62, no. 3, pp. 251-257, 2017.

[17] F. E. Harrell, K. L. Lee Jr., and D. B. Mark, "Multivariable prognostic models: issues in developing models, evaluating assumptions and adequacy, and measuring and reducing errors," Statistics in Medicine, vol. 15, no. 4, pp. 361-387, 1996.

[18] S. Bendifallah, G. Canlorbe, E. Raimond et al., "An external validation study of nomograms designed to predict isolated loco-regional and distant endometrial cancer recurrences: how applicable are they?" British Journal of Cancer, vol. 109, no. 6, pp. 1498-1503, 2013.

[19] M. Momomura, H. Matsumoto, S Umezawa et al., "Histologic grade and peritoneal cytology as prognostic factors in type 1 endometrial cancer," International Journal of Clinical Oncology, vol. 22, no. 3, pp. 533-540, 2017.

[20] S. Sasada, M. Yunokawa, Y. Takehara et al., "Baseline risk of recurrence in stage I-II endometrial carcinoma," Journal of Gynecologic Oncology, vol. 29, no. 1, p. e9, 2018.

[21] T. Tuomi, A. Pasanen, A. Leminen, R. Bützow, and M. Loukovaara, "Prediction of site-specific tumor relapses in patients with stage I-II endometrioid endometrial cancer," International Journal of Gynecological Cancer, vol. 27, no. 5, pp. 923-930, 2017.

[22] K. Matsuo, A. Yabuno, M. S. Hom et al., "Significance of abnormal peritoneal cytology on survival of women with stage I-II endometrioid endometrial cancer," Gynecologic Oncology, vol. 149, no. 2, pp. 301-309, 2018.

[23] B. L. Seagle, A. L. Alexander, T. Lantsman, and S. Shahabi, "Prognosis and treatment of positive peritoneal cytology in early endometrial cancer: matched cohort analyses from the National Cancer Database," American Journal of Obstetrics and Gynecology, vol. 218, no. 3, pp. 329.e1-329.e15, 2018. 
[24] N. Takeshima, H. Nishida, T. Tabata, Y. Hirai, and K. Hasumi, "Positive peritoneal cytology in endometrial cancer: enhancement of other prognostic indicators," Gynecologic Oncology, vol. 82, no. 3, pp. 470-473, 2001.

[25] S. A. Scott, C. van der Zanden, E. Cai, C. E. McGahan, and J. S. Kwon, "Prognostic significance of peritoneal cytology in low-intermediate risk endometrial cancer," Gynecologic Oncology, vol. 145, no. 2, pp. 262-268, 2017.

[26] B. Lee, D. H. Suh, K. Kim, J. H. No, and Y. B. Kim, "Influence of positive peritoneal cytology on prognostic factors and survival in early-stage endometrial cancer: a systematic review and meta-analysis," Japanese Journal of Clinical Oncology, vol. 46, no. 8, pp. 711-717, 2016.

[27] N. R. Abu-Rustum, A. Iasonos, Q Zhou et al., "Is there a therapeutic impact to regional lymphadenectomy in the surgical treatment of endometrial carcinoma?" American Journal of Obstetrics and Gynecology, vol. 198, no. 4, pp. 457.e1-457.e6, 2008.

[28] S. Kondalsamy-Chennakesavan, C. Yu, M. W. Kattan et al., "Nomograms to predict isolated loco-regional or distant recurrence among women with uterine cancer," Gynecologic Oncology, vol. 125, no. 3, pp. 520-525, 2012.

[29] S. Bendifallah, G. Canlorbe, F. Huguet et al., "A risk scoring system to determine recurrence in early-stage type 1 endometrial cancer: a French multicentre study," Annals of Surgical Oncology, vol. 21, no. 13, pp. 4239-4245, 2014. 